\title{
The Influence of Iron-deficiency Anemia during the Pregnancy on Preterm Birth and Birth Weight in South China
}

\author{
LingLing Huang, Gowreesunkur Purvarshi* , SuMei Wang, LinLin Zhong, Hui Tang \\ Department of Obstetrics and Gynecology, The First Affiliated Hospital of GuangXi Medical University, Nanning, GuangXi, China \\ *Corresponding author: 2657316472@qq.com
}

\begin{abstract}
Objective: Iron deficiency anemia (IDA) is very common during pregnancy and it has adverse effects on pregnancy. However, current researches on IDA and adverse pregnancy outcomes have shown inconsistency. The aim of this observational study is to assess in which trimester of pregnancy, IDA carries a greater risk of low birth weight (LBW) infants and preterm birth. Methods: A prospective cohort study was carried out at obstetric department of the First Affiliated Hospital of Guangxi Medical University from January 2014 to December 2014. Venous blood samples of 500 pregnant mothers were collected in first, second and third trimester of pregnancy. Hemoglobin and serum ferritin levels in each trimester of pregnancy were recorded. The mothers were followed till delivery. Gestational weeks at the time of delivery and birth weight of babies were recorded. Results: Our data showed that during pregnancy, more than $70 \%$ of pregnant women suffered iron-deficiency anemia. Compared to the non-IDA group, serum ferritin level was significantly low in second and late trimester of the pregnancy in IDA group ( $\mathrm{p}<0.05)$. Pregnant women who were anemic during the pregnancy were less literate, multigravida, multipara ( $p<0.05)$. According to the logistic regression analysis, Anemia in late trimester was the affected factors to the incidence of low birth weight and preterm birth $(\mathrm{p}<0.05)$ A positive correlation $(r=0.97, \mathrm{p}<0.05)$ between Hb level and birth weight was seen only in late trimester anemic patients. Conclusions: The incidence of preterm deliveries and low birth weight babies were significantly more in mothers who were anemic in the third trimesters of pregnancy. Hemoglobin screening in second trimester is necessary for pregnant women along with correcting the anemia from the second trimester to improve the maternal outcome. Most effective education programs should be performed to the anemic group during pregnancy.
\end{abstract}

Keywords: IDA, ferritin, preterm birth, birth weight, iron therapy

Cite This Article: LingLing Huang, Gowreesunkur Purvarshi, SuMei Wang, LinLin Zhong, and Hui Tang, "The Influence of Iron-deficiency Anemia during the Pregnancy on Preterm Birth and Birth Weight in South China.” Journal of Food and Nutrition Research, vol. 3, no. 9 (2015): 570-574. doi: 10.12691/jfnr-3-9-2.

\section{Introduction}

Iron deficiency anemia is globally the main cause of maternal anemia during pregnancy [1]. Maternal anemia is a characteristic of physiological changes during pregnancy. If iron intake is insufficient or if there are some abnormalities in absorption during this period, iron deficiency anemia can be exacerbated. There is evidence that demands for iron increases during the second and third trimester of pregnancy [2], therefore it is recommended for pregnant women to increase their iron intake during the second and third trimester [3,4]. Lt Manu Tiwari et al studied iron-deficiency anemia in pregnant women in the second and third trimester in India and pointed out that in developing countries, the iron deficiency is prevalent due to inappropriate eating habits and iron supplementation should be prescribed as a routine whereas unlike in developed countries where iron supplementation is prescribed according to serum ferritin and iron levels [5]. During the past decades, most studies concluded that anemia during pregnancy is associated with increased incidence of adverse pregnancy outcomes [6,7]. Therefore, improving the hemoglobin levels during pregnancy will benefit both mother and fetus [8]. However, with the improvement of modern research methods, researchers attempted to re-examine the iron supplementation during pregnancy and the concept of increasing the hemoglobin level during pregnancy. Some randomized controlled trials (RCT) and Meta-analysis showed that, routine iron supplementation is of little use $[9,10]$, even for pregnant women who have anemia during pregnancy [11], and if hemoglobin exceeds a certain level, it can have a negative effect [12]. Studies have shown that high hemoglobin level (120g /L) showed negative outcome in pregnancy African-American adolescents [13].

Current researches on maternal hemoglobin levels and adverse pregnancy outcomes have shown inconsistency. Thus, the present study was constructed to investigate the effect of iron-deficiency anemia in different trimesters and its effect on preterm birth and low birth weight. 


\section{Materials and Methods}

\subsection{Study Design}

A prospective cohort study from January 2014 to December 2015 was carried out at obstetric department of the First Affiliated hospital of Guangxi Medical University. Pregnant women who met the inclusion criteria (see below) and do not fit for exclusion criteria were included. Before enrolment in the study, informed consent was given to the pregnant women. Total 500 pregnant women were included in the study. Obstetrics' information and demographic characteristics of pregnant women were collected. The hemoglobin level, hematocrit level, mean corpuscular volume, mean corpuscular hemoglobin and serum ferritin level were collected in early, middle and late pregnancy. An oral iron supplement (Iron Polysaccharide Complex Capsules, Kremers Urban Pharmaceuticals Inc, USA) was prescribe to the women once diagnosed for lower ferritin level.

\subsection{Study Criteria}

According to current guidelines based on recommendations of World Health Organization (WHO), Iron-deficiency anemia in pregnancy was defined as hemoglobin value less than $110 \mathrm{~g} / \mathrm{L}$ [14] in any trimester during pregnancy

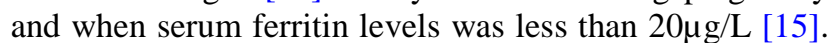
Blood samples that were withdrawn between 11-13 gestational weeks were defined as first trimester pregnancy. Blood samples that were withdrawn between 24-28 gestational weeks were defined as second trimester pregnancy. Blood samples that were withdrawn between 34-35 gestational weeks were defined as late pregnancy. Low birth weight (LBW) was defined as birth weight < $2500 \mathrm{~g}$ and preterm birth as $<37$ weeks of gestation. If a patient had two abnormalities such as LBW and preterm delivery, each was considered an independent outcome, and the subject was included in both categories.

\subsection{Inclusion Criteria}

Pregnant women with singleton pregnancy and negative thalassemia screening.

\subsection{Exclusion Criteria}

Systemic diseases during pregnancy, Blood transfusion at least 3 months before enrolment in the study, smoking and drinking, $\mathrm{MCV}>100, \mathrm{MCH}>34$.

\subsection{Statistical Analysis}

Statistical analyses were conducted using spss 16.0. Quantitative data were recorded as mean \pm standard deviation. Qualitative data were recorded as number of cases or ratio. Comparison of data was done using $t$ test, chi-square test in two groups. ANOVA analysis was used in multi-group comparison. Logistic regression analysis was used to evaluate the influence factors of preterm birth incidence and low birth weight. $\mathrm{P}$ values less than 0.05 were statistically significant.

\section{Results}

\subsection{Demographic Characteristics of Women with Anemia during the Three Trimesters of Pregnancy and Women without Anemia during the Three Trimesters of Pregnancy}

The differences in age and body mass index between the IDA and non-IDA group were not statistically significant $(\mathrm{p}>0.05)$. Compared to the non-anemic group, the level of education of the anemic group was lower than those who did not have anemia during the whole pregnancy and the difference was statistically significant ( $>0.05)$. The anemic group pregnant women also had a higher gravidity and parity compared to the non-anemic group and the difference was also statistically significant $(\mathrm{p}<0.05)$ (Table 1).

Table 1. Demographic characteristics between IDA and non-IDA during the pregnancy

\begin{tabular}{|c|c|c|c|}
\hline & Whole pregnancy IDA (cases) & Whole pregnancy non- IDA (cases) & \\
\hline & 75 & 149 & $\mathrm{P}$ value \\
\hline Age (years) & $27.3 \pm 3.2$ & $25.7 \pm 2.8$ & 0.26 \\
\hline Body mass index on $1^{\text {st }}$ outpatient visit (BMI) & $20.3 \pm 2.4$ & $20.4 \pm 2.5$ & 0.34 \\
\hline \multicolumn{4}{|l|}{ Education level } \\
\hline$<10$ years & $45(60.0 \%)$ & 57 (38.3\%) & \\
\hline$\geq 10$ years & $30(40.0 \%)$ & $92(61.7 \%)$ & 0.03 \\
\hline \multicolumn{4}{|l|}{ Gravidity } \\
\hline 1 & $31(41.3 \%)$ & $75(50.3 \%)$ & \\
\hline 2 & $25(33.3 \%)$ & $51(34.2 \%)$ & \\
\hline$\geq 3$ & $19(25.3 \%)$ & $23(15.4 \%)$ & 0.04 \\
\hline \multicolumn{4}{|l|}{ Parity } \\
\hline Primipara & $62(83.7 \%)$ & 137(92.1\%) & \\
\hline Multipara & $13(17.3 \%)$ & $12(7.9 \%)$ & 0.04 \\
\hline
\end{tabular}

For quantitative variable, ( $\overline{\mathrm{X}} \pm \mathrm{s})$. For qualitative variable, $\mathrm{n}(\%)$

\subsection{Distribution of Iron Deficiency Anemia during Pregnancy}

According to Table 2, in different trimesters of pregnancy, there are a certain percentage of women with iron-deficiency anemia and with low serum ferritin levels.
Among 500 cases, In first trimester there_were 195 cases (39\%) with low level ferritin out of which 185 cases (37\%)developed IDA ; in second trimester there were 295 cases (59\%) with low level ferritin out of which 259 cases (51.8\%) developed IDA; in late trimester 125 cases (25\%) with low level ferritin out of which 116 cases (23.2\%) 
developed IDA. The levels of hemoglobin and serum ferritin in first, second and late pregnancy showed the same tendency with a drop in second trimester and a rise in third trimester but still lower than the first trimester level. 149 (29.8\%) pregnant women had no anemia during the pregnancy and $75(15.0 \%)$ pregnant women had anemia during the pregnancy (Table 1).

Table 2. Distribution of IDA in pregnant women

\begin{tabular}{ccc}
\hline & & Cases (500 cases) \\
\cline { 2 - 3 } & First tri & Second tri \\
\hline No. of IDA pregnant women n (\%) & $185(37.0)$ & $259(51.8)$ \\
No.of low SF pregnant women $\mathrm{T}(\%)$ & $195(39.0)$ & $295(59.0)$ \\
Mean Hb \pm Standard deviation $(\mathrm{g} / \mathrm{dl})$ & $11.2 \pm 1.41$ & $10.9 \pm 0.79$ \\
Mean SF \pm Standard deviation $(\mu \mathrm{g} / \mathrm{dl})$ & $20.32 \pm 4.5$ & $16.13 \pm 5.6$ \\
\hline
\end{tabular}

\subsection{Comparison of Serum Ferritin Levels in Different Trimesters of Pregnancy}

The pregnant women were divided into anemic group and non-anemic group in each trimester of pregnancy and their serum ferritin levels were compared. The difference in serum ferritin levels in anemic and non-anemic group was not statistically significant in first trimester of pregnancy $(p>0.05)$. The difference of serum ferritin was statistically significant in second and third trimester ( $p$ $<0.05$ ) as shown in Table 3.

Table 3. Comparison of serum ferritin levels in different trimesters of pregnancy

\begin{tabular}{cccc}
\hline & IDA group & Non IDA group & P value \\
\hline $1^{\text {ST }}$ tri SF & $19.35 \pm 2.9$ & $21.29 \pm 6.1$ & 0.08 \\
$2^{\text {nd }}$ tri SF & $14.79 \pm 3.7$ & $17.9 \pm 7.4$ & 0.048 \\
$3^{\text {rd }}$ tri SF & $16.42 \pm 4.3$ & $19.7 \pm 6.6$ & 0.049 \\
\hline SF: 5 .
\end{tabular}

SF: serum ferritin. IDA: iron deficiency anemia.

\subsection{IDA in Different Periods of Pregnancy and Its Relation to Birth Weight and Duration of Gestation}

As shown in Table 4, according to anemia in different trimesters of pregnancy, the 500 pregnant women were divided into 7 groups, ANOVA analysis showed that the difference in birth weight and gestational weeks among the groups were not statistically significant $(\mathrm{P}>0.05)$. $\mathrm{T}$ test analysis showed that the significant difference in birth weight between IDA and non-IDA during the pregnancy $(\mathrm{P}<0.05)$ (Table 5).

Table 4. Comparison of birth weight and gestational weeks in groups according to anemia in different trimester

\begin{tabular}{|c|c|c|c|c|c|c|}
\hline Groups & First tri & Second tri & Third tri & Total (500) & Birth weight (g) & Gestation (weeks) \\
\hline 1 & No anemia & No anemia & No anemia & 149 & $2909.66 \pm 212.36$ & $37.78 \pm 1.54$ \\
\hline 2 & Anemia & No anemia & No anemia & 70 & $2899.49 \pm 170.78$ & $37.64 \pm 1.12$ \\
\hline 3 & Anemia & Anemia & No anemia & 40 & $2895.07 \pm 160.44$ & $37.55 \pm 0.98$ \\
\hline 4 & No anemia & Anemia & No Anemia & 125 & $2871.49 \pm 178.40$ & $37.57 \pm 1.33$ \\
\hline 5 & No anemia & No anemia & Anemia & 22 & $2799.49 \pm 228.53$ & $37.04 \pm 1.4$ \\
\hline 6 & No anemia & Anemia & Anemia & 19 & $2773.21 \pm 198.61$ & $37.21 \pm 0.85$ \\
\hline 7 & Anemia & Anemia & Anemia & 75 & $2784.00 \pm 169.00$ & $37.46 \pm 1.44$ \\
\hline $\mathrm{F}$ & & & & & 1.301 & 1.496 \\
\hline $\mathrm{P}$ & & & & & 0.255 & 0.177 \\
\hline
\end{tabular}

For quantitative variable, $(\overline{\mathrm{X}} \pm \mathrm{s})$.

Table 5. Comparison of birth weight and gestational weeks between IDA and non-IDA during the pregnancy

\begin{tabular}{ccccccc}
\hline Groups & First tri & Second tri & Late tri & Total (224) & Birth weight (g) & Gestation (weeks) \\
\hline 1 & Anemia & Anemia & Anemia & 75 & $2784.00 \pm 169.00$ \\
2 & No anemia & No anemia & No anemia & 149 & $2909.66 \pm 212.36 *$ \\
\hline
\end{tabular}

For quantitative variable, $(\overline{\mathrm{X}} \pm \mathrm{s}) . *$ : $\mathrm{P}<0.05$

\subsection{Logistic Regression Analysis the Anemia in Different Trimester to the Incidence of Low Birth Weight Infant and Preterm Birth}

In each trimester of pregnancy, the 500 pregnant women were divided into anemic and non-anemic groups according to the Hb levels. Analysis the effect of anemia in different trimester to the incidence of low birth weight and preterm birth were done by the logistic regression analysis. Anemia in first and second trimester was not entered in formula as the affected factors to the incidence of low birth weight and preterm birth ( $>00.05)$. Anemia in third trimester was entered in formula as the affected factors to the incidence of low birth weight and preterm birth (p <0.05). (Table 6, Table 7).

Table 6. Variables related to preterm delivery and low birth weight infant and their values given in logistic regression

\begin{tabular}{|c|c|c|}
\hline Item & Variable & evaluation \\
\hline The first trimester & $\mathrm{X} 1$ & $1=$ normal, $2=$ IDA \\
\hline The second trimester & $\mathrm{X} 2$ & $1=$ normal, $2=\mathrm{IDA}$ \\
\hline The third trimester & X3 & $1=$ normal, $2=\mathrm{IDA}$ \\
\hline Preterm Birth & Y1 & $1=$ full term, $2=$ =preterm \\
\hline Low birth weight infants & Y2 & 1=normal, $2=$ lower birth weigh \\
\hline
\end{tabular}


Table 7. Analysis of logistic regression on incidence of low birth weight and preterm birth

\begin{tabular}{|c|c|c|c|c|c|c|c|c|c|}
\hline & & \multirow{2}{*}{ B } & \multirow{2}{*}{ S.E, } & \multirow{2}{*}{ Wals } & \multirow{2}{*}{ df } & \multirow{2}{*}{ Sig. } & \multirow{2}{*}{ OR } & \multicolumn{2}{|c|}{ 95\% CI for OR } \\
\hline & & & & & & & & lower & upper \\
\hline \multirow{3}{*}{$\begin{array}{l}\text { Low Birth } \\
\text { Weight }\end{array}$} & $1^{\text {st }}$ trimester & -0.495 & 0.327 & 2.293 & 1 & 0.130 & 0.610 & 0.321 & 1.157 \\
\hline & $2^{\text {nd }}$ trimester & 0.485 & 0.305 & 2.533 & 1 & 0.111 & 1.625 & 0.894 & 2.954 \\
\hline & $3^{\text {rd }}$ trimester & 0.729 & 0.332 & 4.823 & 1 & 0.028 & 2.073 & 1.082 & 3.973 \\
\hline \multirow{3}{*}{$\begin{array}{l}\text { Preterm } \\
\text { Birth }\end{array}$} & $1^{\text {st }}$ trimester & -0.113 & 0.253 & 0.198 & 1 & 0.657 & 0.893 & 0.544 & 1.468 \\
\hline & $2^{\text {nd }}$ trimester & -0.033 & 0.247 & 0.018 & 1 & 0.894 & 0.968 & 0.596 & 1.571 \\
\hline & $3^{\text {rd }}$ trimester & 0.612 & 0.281 & 4.757 & 1 & 0.029 & 1.845 & 1.064 & 3.198 \\
\hline
\end{tabular}

\subsection{Correlation between $\mathrm{Hb}$ Levels and Birth Weight}

The linear correlation between $\mathrm{Hb}$ levels of anemic women in each trimester of pregnancy and birth weight was studied. A positive correlation $(r=0.97, \mathrm{p}<0.05)$ between $\mathrm{Hb}$ level and birth weight was seen only in third trimester anemic patients (116 patients) as shown in the scatter plot below (Figure 1). No correlation between $\mathrm{Hb}$ levels and birth weight was observed in third trimester non-anemic patients (384 patients). There is no correlation between $\mathrm{Hb}$ levels and birth weight was observed in first or second trimester.

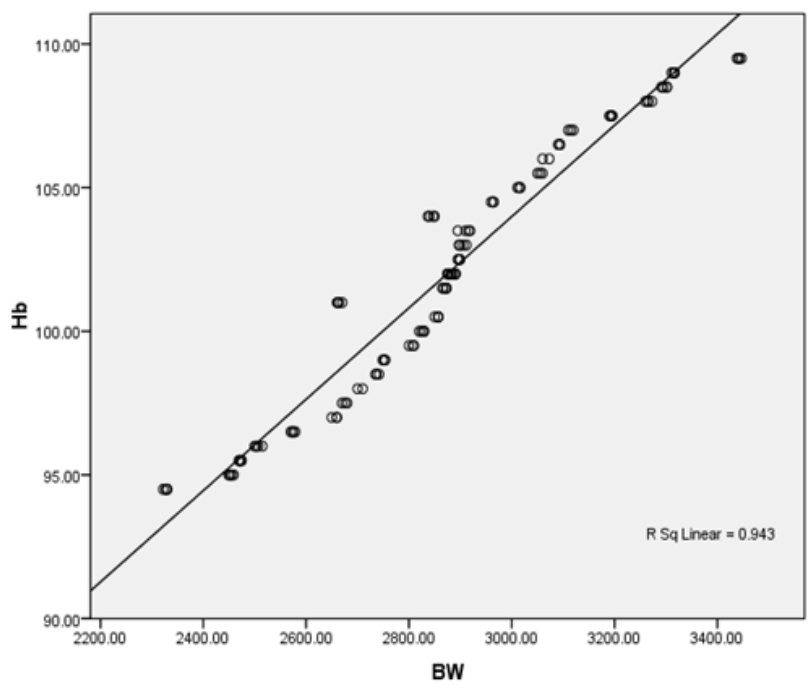

Figure 1. Scatter plot figure of correlation between $\mathrm{Hb}$ and birth weight in third trimester of pregnancy $(r=0.97, \mathrm{p}<0.05)$

\section{Discussion}

Blood volume increases during pregnancy in which plasma volume increases by $30-40 \%$, red blood cells increases by $20-25 \%$.Therefore, the increase in plasma volume is larger than blood volume increase [16] which causes the Pregnancy related physiological anemia. This hemodilution is a physiological change during pregnancy, the low viscosity state of hemodilution helps to increase oxygen transport in placental tissue $[17,18]$. Pregnancy induced hypervolemia has important function, which includes to meet the metabolic demands of the enlarged uterus with its greatly hypertrophied vascular system, to safeguard the mother against the adverse effects of blood loss associated with parturition [19].

The mild hemoglobin decline in pregnancy compared to non pregnancy can be explained by hemodilution and it might get worse by the increased iron requirement for the fetal growth which can cause iron-deficiency anemia. The degree and the incidence of anemia are influenced by gravidity and parity, which were inversely related to education and socioeconomic status [20]. Our study suggests that Education level, gravidity and parity increases the prevalence of IDA in pregnant women. We propose that this might be due to the low iron stored in women who have had more pregnancies and poor response to iron supplements. This observation may indicate that the multigravida subjects require a higher daily dose of iron for a longer time to improve their anemia, raise the awareness of pregnant women about iron deficiency anemia through carrying out the health education.

Our data showed that more than $70 \%$ of pregnant women had iron-deficiency anemia during pregnancy. The highest proportion of anemic women that is more than half of the population were observed during second trimester. The cases of anemia were less and the degree of anemia was reduced in the third trimester, which was relative with the iron supplement from the second trimester. The serum ferritin changes during the three trimesters of pregnancy follows the same tendency. This observation has also been reported in other studies [21].

Anemia would cause the low nutrients and decreased oxygen carried capacity, which cause the placenta oxygen and nutrients cannot meet the need of fetal growth and development, lead to fetal growth restriction. In addition, Hypoxia caused by Iron deficiency anemia may induces maternal and fetal stress, and the release of the adrenal cortex hormone releasing hormone (CRH), and cause a series of downstream effects, including regulating cell factor inhibits fetal weight gain, stimulating the release of prostaglandin F2a and promote uterine contraction [22]. Although it is known that iron-deficiency anemia in pregnant women can cause adverse outcomes such as increased risk of delivery of premature and LBW babies, IUD, low APGAR score at $1 \mathrm{~min}$ and perinatal mortality. Deaths were commonly due to prematurity and sepsis [23]. However, the impacts of IDA are still controversial in different periods. Studies have indicated that the low hemoglobin in early pregnancy can cause adverse pregnancy outcome [24]. A retrospective study showed that there was no association between early pregnancy and low birth weight [25]. A number of studies have reported a significant association between low birth weight, gestational age and anemia in late trimester [26,27].

According to our study, ANOVA study showed that there was no difference in comparison of birth weight and gestational weeks in groups according to anemia in different trimester. Even though Table 5 showed the birth weight was bigger in the non-IDA group during the pregnancy, from the specific data, we found that birth weight was in the clinical normal range. These differences 
cannot be used as the evidence to guide the iron supplement management. According to the logistic analysis, the incidence of low birth weight babies and preterm births was higher in pregnant women who had anemia in third trimester than non- anemic group. The correlation analysis showed a positive correlation between hemoglobin level and birth weight only in late trimester of pregnancy but not in early or mid trimester. This proves that when $\mathrm{Hb}$ level reaches a certain level, its influence on birth weight and reaches a platform level, but further increasing the $\mathrm{Hb}$ level will not increase the birth weight. Our data also suggested that serum ferritin levels also showed significant decline during pregnancy. In addition to implementing more iron-rich source food in the diet during pregnancy, iron supplementation should be prescribed during middle and late pregnancy. It should be noted that iron supplementation should not be taken excessively because it is still uncertain for the side effect of iron overdose. Therefore, iron supplementation should be based on hematological parameters.

In summary, clinicians should be aware about iron deficiency anemia during pregnancy especially during the second and third trimester of pregnancy in order to improve perinatal outcomes. Because of the limited number of cases in this study, we were not able to analyze the effect of high level of hemoglobin levels during pregnancy on perinatal outcomes. It is still not clear whether high hemoglobin levels can cause adverse perinatal outcomes. In order to avoid excessive iron-intake, regular monitoring of hemoglobin levels, serum ferritin levels and other hematological parameters are essential.

\section{Acknowledgements}

Acknowledgement for financial support, the Medical and Health Foundation of GuangXi, China ( Z2015509).

\section{Author Disclosure Statement}

\section{No competing financial interests exist.}

\section{References}

[1] Cuervo LG, Mahomed K. Treatments for iron deficiency anaemia in pregnancy. Cochrane Database Syst Rev. 2001. (2): CD003094.

[2] Allen LH. Pregnancy and iron deficiency: unresolved issues. Nutr Rev. 1997. 55(4): 91-101.

[3] Spinillo A, Capuzzo E, Piazzi G, Nicola S, Colonna L, Iasci A. Maternal high-risk factors and severity of growth deficit in small for gestational age infants. Early Hum Dev. 1994. 38(1): 35-43.

[4] Malhotra M, Sharma JB, Batra S, Sharma S, Murthy NS, Arora R. Maternal and perinatal outcome in varying degrees of anemia. Int J Gynaecol Obstet. 2002. 79(2): 93-100.

[5] Tiwari M, Kotwal J, Kotwal A, Mishra P, Dutta V, Chopra S. Correlation of haemoglobin and red cell indices with serum ferritin in Indian women in second and third trimester of pregnancy. Med J Armed Forces India. 2013. 69(1): 31-6.
[6] Rusia U, Madan N, Agarwal N, Sikka M, Sood SK. Effect of maternal iron deficiency anaemia on foetal outcome. Indian $\mathrm{J}$ Pathol Microbiol. 1995. 38(3): 273-9.

[7] Levy A, Fraser D, Katz M, Mazor M, Sheiner E. Maternal anemia during pregnancy is an independent risk factor for low birthweight and preterm delivery. Eur J Obstet Gynecol Reprod Biol. 2005. 122(2): 182-6.

[8] Haider BA, Olofin I, Wang M, Spiegelman D, Ezzati M, Fawzi WW. Anaemia, prenatal iron use, and risk of adverse pregnancy outcomes: systematic review and meta-analysis. BMJ. 2013. 346: f3443.

[9] Hemminki E, Merilainen J. Long-term follow-up of mothers and their infants in a randomized trial on iron prophylaxis during pregnancy. Am J Obstet Gynecol. 1995. 173(1): 205-9.

[10] Pena-Rosas JP, Viteri FE. Effects of routine oral iron supplementation with or without folic acid for women during pregnancy. Cochrane Database Syst Rev. 2006. (3): CD004736.

[11] Allen LH. Anemia and iron deficiency: effects on pregnancy outcome. Am J Clin Nutr. 2000. 71(5 Suppl): 1280S-4S.

[12] Koller O, Sandvei R, Sagen N. High hemoglobin levels during pregnancy and fetal risk. Int J Gynaecol Obstet. 1980. 18(1): 53-6.

[13] Chang SC, O'Brien KO, Nathanson MS, Mancini J, Witter FR. Hemoglobin concentrations influence birth outcomes in pregnant African-American adolescents. J Nutr. 2003. 133(7): 2348-55.

[14] McLean E, Cogswell M, Egli I, Wojdyla D, de Benoist B. Worldwide prevalence of anaemia, WHO Vitamin and Mineral Nutrition Information System, 1993-2005. Public Health Nutr. 2009. 12(4): 444-54.

[15] ACOG Practice Bulletin No. 95: anemia in pregnancy. Obstet Gynecol. 2008. 112(1): 201-7.

[16] Clark SL, Cotton DB, Lee W, et al. Central hemodynamic assessment of normal term pregnancy. Am J Obstet Gynecol. 1989. 161(6 Pt 1): 1439-42.

[17] Molendijk L, Malburg I, Kopecky P. [Doppler ultrasound studies in placental insufficiency as an indication of the effectiveness of hemodilution therapy]. Z Geburtshilfe Neonatol. 1995. 199(1): 1822.

[18] Peeters LL, Verkeste CM, Saxena PR, Wallenburg HC. Relationship between maternal hemodynamics and hematocrit and hemodynamic effects of isovolemic hemodilution and hemoconcentration in the awake late-pregnant guinea pig. Pediatr Res. 1987. 21(6): 584-9.

[19] Moin A, Lassi ZS. Can routine screening and iron supplementation for iron deficiency anemia in nonsymptomatic pregnant women improve maternal and infant health outcomes. J Family Med Prim Care. 2015. 4(3): 333-4.

[20] Al-Farsi YM, Brooks DR, Werler MM, Cabral HJ, Al-Shafei MA, Wallenburg HC. Effect of high parity on occurrence of anemia in pregnancy: a cohort study. BMC Pregnancy Childbirth. 2011. 11: 7.

[21] Kalaivani K. Prevalence \& consequences of anaemia in pregnancy. Indian J Med Res. 2009. 130(5): 627-33.

[22] Allen LH. Biological mechanisms that might underlie iron's effects on fetal growth and preterm birth. J Nutr. 2001. 131(2S-2): 581S-589S.

[23] Lone FW, Qureshi RN, Emanuel F. Maternal anaemia and its impact on perinatal outcome. Trop Med Int Health. 2004. 9(4): 486-90.

[24] Hamalainen H, Hakkarainen K, Heinonen S. Anaemia in the first but not in the second or third trimester is a risk factor for low birth weight. Clin Nutr. 2003. 22(3): 271-5.

[25] Blankson ML, Goldenberg RL, Cutter G, Cliver SP. The relationship between maternal hematocrit and pregnancy outcome: black-white differences. J Natl Med Assoc. 1993. 85(2): 130-4.

[26] Lone FW, Qureshi RN, Emanuel F. Maternal anaemia and its impact on perinatal outcome. Trop Med Int Health. 2004. 9(4): 486-90.

[27] Levy A, Fraser D, Katz M, Mazor M, Sheiner E. Maternal anemia during pregnancy is an independent risk factor for low birthweight and preterm delivery. Eur J Obstet Gynecol Reprod Biol. 2005. 122(2): 182-6. 